\section{Fatores associados à cárie dentária em pré- escolares do Recife, Pernambuco, Brasil}

\author{
Factors associated with dental caries in preschoolers \\ in Recife, Pernambuco State, Brazil
}

\author{
${ }^{1}$ Centro de Ciências da \\ Saúde, Universidade Federal \\ de Pernambuco, Recife, \\ Brasil. \\ 2 Secretaria de Saúde do \\ Estado de Pernambuco, \\ Recife, Brasil. \\ 3 Centro de Pesquisas Aggeu \\ Magalhães, Fundação \\ Oswaldo Cruz, Recife, Brasil. \\ Correspondência \\ M. M. D. C. Melo \\ Departamento de Clínica \\ e Odontologia Preventiva, \\ Centro de Ciências da Saúde, \\ Universidade Federal de \\ Pernambuco. \\ Av. Professor Moraes Rego s/n \\ Recife, PE 56670-901, Brasil. \\ marciamdcm@hotmail.com
}

\section{Abstract}

To investigate factors associated with dental caries in deciduous dentition, a nested case-control study was conducted in a prevalence survey of preschool children $(n=1,690)$ aged 18 to 36 months and 5 years of age, treated under the Family Health Program in Recife, Pernambuco State, Brazil, in 2006. Cases were children with dmft $\geq$ 1 and controls with $d m f t=0$. The independent variables were analyzed in blocks: demographic, environmental, family structure, health care, and dental services use. OR and 95\%CI were estimated. Variables associated with the outcome at $p \leq 0.20$ in the univariate analysis were included in a multivariate logistic regression model. The cutoff for remaining in the final model was $p \leq$ 0.10. Larger households (more members), lack of running supply, time of residence, maternal (or caregiver's) schooling, attendance in public preschool, demand for dental services, and sugar intake patterns were associated factors. Variables related to social context were the main predictors of caries, thus highlighting the need for comprehensive measures to address other common childhood health problems.

Dental Caries; Preeschool Child; Social Conditions; Dental Health Services
Marcia Maria Dantas Cabral de Melo 1,2 Wayner Vieira de Souza 3 Maria Luiza Carvalho de Lima ${ }^{3}$ Cynthia Braga 3

\section{Introdução}

O ataque de cárie dentária durante a infância é causa frequente de dor, desconforto e dificuldades de alimentação, que comprometem a qualidade de vida e o desenvolvimento biopsicossocial infantil 1 .

Estudos nacionais e internacionais têm constatado uma expressiva redução nos índices de cárie na população escolar 2,3 , porém taxas de prevalência e incidência relativamente mais elevadas ainda são observadas em crianças em idade pré-escolar, particularmente nos grupos com precárias condições de vida 4,5,6. Diante disso, além dos estudos que investigam variáveis biológicas e comportamentais associadas à cárie dentária pré-escolar, tem-se observado um interesse crescente pela investigação dos determinantes socioambientais envolvidos na ocorrência do agravo nessa população 6,7,8,9.

Dados do último levantamento epidemiológico de abrangência nacional realizado no Brasil mostraram elevados índices de cárie na população pré-escolar, além de um padrão heterogêneo de distribuição da sua ocorrência entre regiões do país, sendo as localidades menos favorecidas as mais afetadas 10. Esse perfil epidemiológico reflete, em parte, o processo de conformação das políticas de saúde bucal no país, que historicamente priorizou a assistência odontológica, notadamente para os escolares, em detrimento de um modelo de atenção regido 
pelos conceitos da universalidade e integralidade à saúde bucal 2 .

Nos últimos anos, visando à promoção da equidade em saúde e à melhoria da qualidade de vida da população, o Ministério da Saúde 11 tem desenvolvido políticas públicas de saúde bucal amplas, com foco no cuidado integral à saúde para todas as idades. A partir de 2001, é iniciada a expansão contínua do setor integrada às proposições da Estratégia Saúde da Família (ESF). Entretanto, ainda são verificadas dificuldades de implantar uma atenção à saúde bucal ampla e efetivamente universal 12.

Nessa circunstância, é pertinente a investigação de fatores relacionados à cárie dentária em pré-escolares, no âmbito da atenção primária à saúde, a fim de fornecer informações contextualizadas sobre os principais fatores envolvidos na produção desse agravo. Além disso, o estudo sobre os fatores envolvidos na ocorrência da cárie nessa época da vida reveste-se de importância, já que a experiência de cárie na dentição decídua é um preditor do agravo na dentição permanente 13 .

Embora, nesse contexto, existam estudos sobre as condições de saúde bucal pré-escolar, ainda são escassas as pesquisas publicadas no país após a inserção da saúde bucal na ESF 14,15. Esse fato explica-se, em parte, pela recente inclusão, de maneira programada, dos pré-escolares nos planos e estratégias de ações de saúde bucal na atenção primária à saúde do país 16 .

Com esse intuito, um inquérito de cárie dentária em 2.020 pré-escolares assistidos pela ESF, realizado na cidade do Recife, Pernambuco, Brasil, em 2006 17, encontrou, aproximadamente, prevalências de $29,7 \%$ em crianças com idade entre 18 e 36 meses e 63,8\% aos cinco anos. Esses valores são considerados elevados de acordo com referências nacionais e internacionais 10,18 .

Neste artigo, foram identificados os fatores associados à experiência de cárie dentária na população sob investigação.

\section{Métodos}

Trata-se de um estudo de caso-controle aninhado a um inquérito de prevalência de cárie dentária na dentição decídua, realizado no ano de 2006, em uma população de crianças nas faixas etárias entre 18 e 36 meses e cinco anos, cadastradas nas unidades de saúde família (USF) da ESF de dois Distritos Sanitários (DS) do Município do Recife: o DS II e DS IV 17.

A cidade do Recife, capital do Estado de Pernambuco, possui população de 1.561 .659 habitantes, extensão territorial de $218,75 \mathrm{~km}^{2}$, sendo dividida em 94 bairros, agrupados em seis Regiões Político-Administrativas (RPA), cujos limites geográficos coincidem com as áreas dos DS. Por sua vez, cada RPA é dividida em três microrregiões (Instituto Brasileiro de Geografia e Estatística. Cidades@.http://www.ibge.gov.br/cidadesat/ topwindow.htm, acessado em 16/Fev/2010) 19.

No DS II, localizado na zona norte da cidade, residem $20 \%$ da população do município; cerca de $70 \%$ dos moradores habita em áreas com precária infraestrutura, e $28 \%$ das famílias têm renda inferior a um salário mínimo. No DS IV, zona oeste da cidade, residem $18 \%$ da população; aproximadamente $50 \%$ das famílias vivem em assentamentos de baixa renda, e cerca de $20 \%$ dos moradores desse DS têm renda inferior a um salário mínimo. Quanto à rede pública de saúde, em 2006, o DS II dispunha de 15 USF, as quais respondiam pela assistência à saúde de 29.118 famílias cadastradas, enquanto o DS IV contava com 16 USF para um total de 25.070 famílias cadastradas, correspondendo a uma cobertura de aproximadamente $50 \%$ da população residente em ambos os distritos 19. Vale salientar que essas famílias acompanhadas pela ESF nos DS estudados só foram contempladas com a assistência à saúde bucal, realizada por meio das equipes de saúde bucal (ESB), a partir de 2001.

Para o cálculo do tamanho da amostra do inquérito de prevalência, foram utilizados os parâmetros de $27 \%$ de prevalência de cárie aos 18 36 meses e de $65 \%$ para a idade de cinco anos, baseados em informações para o Nordeste do último levantamento nacional ${ }^{10}$. Admitindo-se um erro amostral de 5\% e 6,5\% nas respectivas idades, um grau de $95 \%$ de confiança e efeito de desenho igual a 1, estimou-se um tamanho de amostra de 3.000 crianças (sendo $\mathrm{n}=1.800$ aos 18-36 meses; $\mathrm{n}=1.200$ aos 5 anos, para o conjunto dos dois distritos). A alocação da amostra em cada DS foi realizada por cotas iguais de crianças em cada faixa etária $(\mathrm{n}=1.500 \mathrm{em}$ cada distrito: 18-36 meses, $\mathrm{n}=900 ; 5$ anos, $\mathrm{n}=$ 600). Então, em cada DS, 9.480 famílias foram selecionadas proporcionalmente ao número de famílias inscritas nas USF de cada DS. Em seguida, foram selecionadas nessas famílias todas as crianças que estivessem nas idades previstas pelo estudo. Participaram do inquérito 2.020 indivíduos dos dois DS (1.415, de 18-36 meses; 605 , de 5 anos), o que representou uma taxa global de participação de $67,3 \% 17$.

Crianças que apresentassem um índice ceo-d (número de dentes cariados, perdidos e obturados na dentição decídua) maior ou igual a um (ceo- $\mathrm{d} \geq 1$ ) foram consideradas caso, ao passo que aquelas com o índice ceo-d igual a zero $($ ceo- $\mathrm{d}=0)$ foram consideradas controle. 
Neste estudo, os casos (ceo- $d \geq 1$ ) e os controles $($ ceo- $\mathrm{d}=0$ ) foram selecionados dos indivíduos identificados no inquérito de prevalência 17. Para tal, realizou-se um processo de amostragem visando a reproduzir os percentuais de prevalência encontrados no inquérito, além de restituir a correta proporcionalidade entre o número de indivíduos examinados e o número de famílias cadastradas em cada DS. Sendo assim, para seleção da amostra aleatória simples de casos e controles para cada grupo etário, foi utilizada uma população de 408 casos (29\%) e 1.007 controles, no grupo etário de 18-36 meses, e de 374 casos (62\%) e 231 controles, no grupo de cinco anos de idade, encontrados no referido inquérito.

Os dados utilizados neste estudo foram obtidos por intermédio das entrevistas estruturadas (questionários) e exames epidemiológicos para medição do índice ceo-d, realizados pelo citado inquérito 17. Os códigos e critérios adotados seguiram as orientações da Organização Mundial da Saúde (OMS) 20; o controle de qualidade dos dados foi realizado pela análise de reprodutibilidade das observações efetuadas pelos examinadores e pela revisão e pré-teste do questionário de entrevista.

A coleta de dados foi realizada nos consultórios odontológicos das USF, após os pais ou responsáveis pelas crianças terem assinado o Termo de Consentimento Livre e Esclarecido. Os exames foram realizados em ambiente sob luz natural, precedido de limpeza dos dentes com gaze umedecida em água, sem auxílio de secagem ou radiografia. Empregou-se instrumental de exame indicado para levantamentos epidemiológicos de cárie 20 . As crianças foram examinadas em decúbito dorsal, na cadeira odontológica colocada em posição horizontal. O examinador ficou sentado e o anotador, em pé e de frente para o profissional, sendo responsável pela anotação dos registros na ficha de exame e pela garantia da biossegurança. Os responsáveis pelas crianças, quando necessário, acompanharam-nas na realização dos exames, após o que, as crianças com necessidades odontológicas foram agendadas para tratamento 17 .

Participaram da coleta de dados 31 examinadores e 31 auxiliares pertencentes às ESB das USF incluídas neste estudo. Eles receberam um treinamento e calibração de 18 horas. Durante o desenvolvimento do inquérito, realizaram também exames em duplicata em $10 \%$ da amostra. Os resultados da calibração foram observados por meio do percentual geral de concordância (PGC) e do teste estatístico de kappa. Os valores gerais obtidos alcançaram escores de confiabilidade recomendados para levantamentos epidemiológicos de cárie dentária 20,21 [PGC (\%) e kappa (IC95\%), respectivamente, interexaminadores: 95,6; 0,73 $(0,71-0,75)$ e intraexaminadores: 99,8; 0,99 (0,91-1,00)] 17.

Com base nas entrevistas, foram obtidas informações sobre as variáveis independentes; estas foram agrupadas nos seguintes blocos de variáveis: (i) sociodemográficas e ambientais (sexo, escolaridade materna, ocupação do pai, ocupação da mãe, moradores por domicílio, número de pessoas por quarto de dormir, origem da água do domicílio, fornecimento de água pela rede geral, canalização interna, distrito sanitário de residência, tempo de moradia na área, faixa etária); (ii) estrutura familiar (com quem a criança mora, expediente em que a mãe trabalha, número de irmãos que moram na mesma casa, ordem de nascimento); (iii) cuidados com a saúde bucal (cuidador diário da criança, escolaridade do cuidador, ingestão de doces entre as refeições, aleitamento materno atualmente, mamadeira noturna presente, periodicidade da escovação, frequência diária de escovação, uso de pasta dental, quem realiza escovação) e (iv) uso de serviços de educação e saúde bucal (criança frequenta creche ou pré-escola, tipo de creche ou pré-escola, procura pelas ações de saúde bucal nas USF, razão da procura, utilização de outros serviços de saúde bucal).

A análise foi realizada separadamente para cada grupo etário, tendo em vista que o número de elementos dentários e a maturidade do órgão dental 8 são menores nas crianças entre 18-36 meses. Esse fator diferencia substancialmente os dois estratos, porém engloba a amostra total de ambos os DS.

Efetuou-se, inicialmente, a análise univariada, calculando-se o odds ratio (OR) bruto e seus respectivos IC95\% para cada bloco de variáveis. As variáveis independentes que se associaram ao desfecho a um nível de significância de $\mathrm{p} \leq$ 0,20 foram incluídas no modelo de regressão logística multivariada, de seu respectivo bloco. As variáveis que apresentaram associação estatisticamente significante com o desfecho nos modelos de regressão múltipla, dentro de cada bloco, foram reunidas em um novo modelo multivariado para obtenção do modelo final. Utilizou-se o método de seleção de variáveis backward stepwise baseado na avaliação do logaritmo da razão de verossimilhança, tomando-se como critério de permanência da variável no modelo final um nível de significância $\mathrm{p} \leq 0,10$. As análises foram realizadas utilizando-se o programa estatístico SPSS na versão 8.0 (SPSS Inc., Chicago, Estados Unidos).

O projeto de pesquisa foi aprovado pelo Comitê de Ética em Pesquisas do Centro de Pesquisas Aggeu Magalhães, unidade da Fundação 
Osvaldo Cruz, Pernambuco (CEP/CPqAM/Fiocruz), sob o número 21/06.

\section{Resultados}

Realizada a seleção da amostra de casos e controles, obteve-se um total de 1.690 crianças participantes, que foram incluídas no estudo, sendo 907 do DS II e 783 do DS IV. Para o conjunto das áreas, foram então selecionadas 353 crianças classificadas como casos e 858, como controles, na faixa etária de 18-36 meses; 301 classificadas como casos e 178, como controles, na idade de cinco anos.

A Tabela 1 apresenta os resultados da análise univariada entre os blocos de variáveis (i) e (ii) (sociodemográficas e ambientais e estrutura familiar) e a presença de ceo-d $\geq 1 \mathrm{em}$ ambas as faixas etárias.

No primeiro bloco, baixa escolaridade materna $(<8$ anos de estudos: $18-36$ meses, $\mathrm{p}=0,0063$, e 5 anos, $\mathrm{p}<0,000$; 8-10 anos de estudos: 18-36 meses, $\mathrm{p}=0,0179$, e 5 anos, $\mathrm{p}=0,0041$ ); moradia em domicílios com seis ou mais moradores (1836 meses, $\mathrm{p}=0,0188$; 5 anos, $\mathrm{p}<0,0001$ ) e com quatro a cinco moradores na idade de cinco anos ( $\mathrm{p}=0,0009$ ), $>3$ pessoas por quarto (18-36 meses, $\mathrm{p}<0,0001 ; 5$ anos, $\mathrm{p}=0,0002)$; tempo de moradia na área de três ou mais anos (18-36 meses, p < 0,0001; 5 anos, $p=0,0095$ ); e faixa etária entre 3136 meses ( $p=0,0001$ ) estiveram estatisticamente associados à experiência de cárie dentária nos grupos etários investigados. Já para a faixa etária entre 18-36 meses, o fato de a origem da água do domicílio ser de poço/nascente $(\mathrm{p}=0,0139)$ apresentou-se negativamente associado a um ceo-d $\geq 1$.

Quanto ao bloco relativo à estrutura familiar, contrariamente ao observado nas crianças de cinco anos, mães que não trabalham fora constituíram-se em fator associado para a cárie no grupo entre $18-36$ meses $(p=00239)$. No que se refere a morar com três ou mais irmãos em ambas as idades (18-36 meses, $\mathrm{p}=0$,0001; 5 anos, $\mathrm{p}<0,0001$ ), ou com dois irmãos (5 anos, $\mathrm{p}=0,0277$ ), e a ser primeiro (5 anos, $\mathrm{p}=0,0090) \mathrm{e}$ intermediário em ambas as idades (18-36 meses, $\mathrm{p}=0,0208 ; 5$ anos, $\mathrm{p}=0,0012$ ), conforme a ordem de nascimento da criança, foram fatores que se associaram à cárie.

Na Tabela 2, são apresentados os resultados da análise univariada entre os blocos de variáveis (iii) e (iv) (cuidados com a saúde bucal e uso de serviços de educação e saúde bucal) e um ceo- $d \geq 1$, nas faixas etárias estudadas.

No bloco de variáveis relativas aos cuidados com a saúde bucal, a baixa escolaridade do cui- dador ( $<8$ anos de estudo: 5 anos, $\mathrm{p} \leq 0,0001$; 8-10 anos de estudo: 18-36 meses, $\mathrm{p}=0,0185,5$ anos, $\mathrm{p}=0,0254$ ) e a ingestão diária de doces entre as refeições (18-36 meses, $\mathrm{p}<0,0001 ; 5$ anos, $\mathrm{p}<0,0001$ ) foram fatores associados à cárie nas idades estudadas. Quanto ao uso de serviços de educação e saúde bucal, a busca por tratamento (18-36 meses, $\mathrm{p}<0,0004$; 5 anos, $\mathrm{p}<0,0001$ ) esteve fortemente associada à cárie dentária, em ambos os grupos etários. Por sua vez, o atendimento de emergência associou-se apenas com a idade de cinco anos ( $\mathrm{p}<0,0004)$. Não frequentar creches constituiu um fator associado à ocorrência de cárie aos 18-36 meses ( $\mathrm{p}=0$,0153). Frequentar creche ou pré-escola pública mostrou associação para a faixa etária de 18-36 meses $(\mathrm{p}=0,0079)$ e para a idade de cinco anos $(p=0,0001)$. Finalmente, frequentar creche ou pré-escola filantrópica associou-se apenas com a idade de 5 anos $(\mathrm{p}=0,0151)$. A não procura pelas ações de saúde bucal oferecidas na USF apresentou-se negativamente associada com a idade de cinco anos ( $p=0,0050$ ), e o uso de mamadeira noturna, com a faixa etária de 18-36 meses ( $\mathrm{p}<0,0001)$.

As Tabelas 3 e 4 apresentam, para os grupos etários de 18-36 meses e cinco anos, respectivamente, os resultados da análise de regressão múltipla de cada bloco (ORajustada 1) e do modelo final (ORajustada 2). Este incluiu todas as variáveis com ORajustada 1 significante na análise por blocos.

No modelo final ajustado para o grupo etário de 18-36 meses, as variáveis que apresentaram associações positivas com a experiência de cárie foram: ser de faixa etária mais elevada (OR = 2,29; IC95\%: 1,32-3,97), residir em domicílios com mais de três moradores por quarto $(\mathrm{OR}=1,68$; IC95\%: 1,23-2,29), ter mais de três irmãos que moram na mesma casa $(\mathrm{OR}=1,71$; IC95\%: 1,10-2,66), ingerir diariamente doces entre as refeições (OR = 2,81; IC95\%: 1,66-4,73) e buscar os serviços para tratamento $(\mathrm{OR}=2,05$; IC95\%: 1,20-3,50). Associações negativas foram encontradas para utilizar água de poço/nascente $(\mathrm{OR}=0,36$; IC95\%: 0,15-0,88) e uso de mamadeira noturna $(\mathrm{OR}=0,68$; IC95\%: 0,51-0,90). Por ter contribuído significativamente para o ajuste do modelo, a variável número de anos de residência na área foi mantida no modelo final, embora, individualmente, nenhuma categoria tenha se associado estatisticamente com a experiência de cárie dentária (Tabela 3).

Na faixa etária de cinco anos, permaneceram no modelo final residir em domicílios com seis ou mais moradores (OR = 3,46; IC95\%: 1,70 $7,00)$, baixa escolaridade do cuidador $(<8$ anos de estudo: OR = 2,95; IC95\%: 1,53-5,67), ingerir diariamente doces entre as refeições $(\mathrm{OR}=3,85$; IC95\%: 1,71-8,63), frequentar pré-escola pública 
Análise univariada da associação entre a experiência de cárie dentária e variáveis sociodemográficas e ambientais e da estrutura familiar, nas faixas etárias de 18-36 meses e 5 anos de idade. Recife, Pernambuco, Brasil, 2006.

\begin{tabular}{|c|c|c|c|c|c|c|c|c|c|c|}
\hline \multirow[t]{4}{*}{ Variáveis/Categorias } & \multicolumn{10}{|c|}{ Faixa etária } \\
\hline & \multicolumn{5}{|c|}{ 18-36 meses } & \multicolumn{5}{|c|}{5 anos } \\
\hline & \multirow[t]{2}{*}{ Total } & \multicolumn{2}{|c|}{$\begin{array}{l}\text { Casos } \\
\text { ceo-d } \geq 1\end{array}$} & \multirow[t]{2}{*}{ OR } & \multirow[t]{2}{*}{ IC95\% } & \multirow[t]{2}{*}{ Total } & \multicolumn{2}{|c|}{$\begin{array}{c}\text { Casos } \\
\text { ceo-d } \geq 1\end{array}$} & \multirow[t]{2}{*}{ OR } & \multirow[t]{2}{*}{ IC95\% } \\
\hline & & $\mathrm{n}$ & $\%$ & & & & $n$ & $\%$ & & \\
\hline \multicolumn{11}{|l|}{ Bloco socioambiental } \\
\hline \multicolumn{11}{|l|}{ Sexo } \\
\hline Feminino & 608 & 172 & 48,7 & 1,00 & - & 226 & 145 & 48,2 & 1,00 & - \\
\hline Masculino & 603 & 181 & 51,3 & 1,09 & $0,84-1,40$ & 253 & 156 & 51,8 & 0,90 & $0,61-1,33$ \\
\hline \multicolumn{11}{|c|}{$\begin{array}{l}\text { Escolaridade materna (anos de } \\
\text { estudo) }\end{array}$} \\
\hline$<8$ & 592 & 184 & 53,8 & 1,62 & $1,13-2,32$ & 254 & 186 & 63,1 & 3,91 & $2,35-6,52$ \\
\hline $8-10$ & 337 & 103 & 30,1 & 1,58 & $1,06-2,34$ & 110 & 67 & 22,7 & 2,23 & $1,24-4,01$ \\
\hline 11 ou mais & 252 & 55 & 16,1 & 1,00 & - & 102 & 42 & 14,2 & 1,00 & - \\
\hline \multicolumn{11}{|l|}{ Ocupação da mãe } \\
\hline Especializada & 25 & 4 & 1,2 & 1,00 & - & 11 & 8 & 2,7 & 1,00 & - \\
\hline Semiespecializada & 36 & 8 & 2,3 & 1,50 & $0,34-6,94$ & 15 & 7 & 2,4 & 0,33 & $0,04-2,26$ \\
\hline Não especializada & 276 & 82 & 24,0 & 2,22 & $0,69-7,90$ & 110 & 66 & 22,5 & 0,56 & $0,11-2,52$ \\
\hline Não ocupada & 856 & 248 & 72,5 & 2,14 & $0,69-7,44$ & 326 & 212 & 72,4 & 0,70 & $0,14-2,96$ \\
\hline \multicolumn{11}{|l|}{ Ocupação do pai } \\
\hline Especializado & 37 & 12 & 3,8 & 1,00 & - & 13 & 8 & 3,0 & 1,00 & - \\
\hline Semiespecializado & 192 & 53 & 17,0 & 0,79 & $0,35-1,82$ & 81 & 42 & 15,8 & 0,67 & $0,17-2,54$ \\
\hline Não especializado & 664 & 197 & 63,2 & 0,88 & $0,41-1,90$ & 245 & 166 & 62,4 & 1,31 & $0,36-4,61$ \\
\hline Não ocupado & 189 & 50 & 16,0 & 0,75 & $0,33-1,72$ & 82 & 50 & 18,8 & 0,98 & $0,25-3,70$ \\
\hline \multicolumn{11}{|l|}{ Moradores por domicílio } \\
\hline Até 3 & 266 & 69 & 19,5 & 1,00 & - & 90 & 37 & 12,4 & 1,00 & - \\
\hline $4-5$ & 581 & 159 & 45,1 & 1,08 & $0,76-1,52$ & 227 & 140 & 47,0 & 2,31 & $1,36-3,91$ \\
\hline 6 ou mais & 360 & 125 & 35,4 & 1,52 & $1,05-2,19$ & 159 & 121 & 40,6 & 4,53 & $2,52-8,28$ \\
\hline \multicolumn{11}{|c|}{ Número de pessoas por quarto } \\
\hline \multicolumn{11}{|c|}{ de dormir } \\
\hline Até 3 & 813 & 206 & 58,4 & 1,00 & - & 302 & 170 & 57,0 & 1,00 & - \\
\hline Mais de 3 & 394 & 147 & 41,6 & 1,75 & $1,34-2,29$ & 174 & 128 & 43,0 & 2,16 & $1,41-3,31$ \\
\hline \multicolumn{11}{|c|}{ Origem da água do domicílio } \\
\hline Rede geral & 1.138 & 338 & 95,7 & 1,00 & - & 445 & 279 & 93,0 & 1,00 & - \\
\hline Poço/Nascente & 51 & 7 & 2,0 & 0,38 & $0,15-0,88$ & 24 & 15 & 5,0 & 0,99 & $0,40-2,52$ \\
\hline Outra situação & 21 & 8 & 2,3 & 1,46 & $0,55-3,80$ & 9 & 6 & 2,0 & 1,19 & $0,26-6,09$ \\
\hline Fornecimento de água pela & & & & & & & & & & \\
\hline rede geral & & & & & & & & & & \\
\hline Diária, sem interrupção & 244 & 72 & 20,6 & 1,00 & - & 84 & 61 & 20,7 & 1,00 & - \\
\hline Diária, com interrupção & 221 & 61 & 17,5 & 0,91 & $0,60-1,39$ & 103 & 64 & 21,7 & 0,62 & $0,32-1,21$ \\
\hline Sem frequência diária & 654 & 201 & 57,6 & 1,06 & $0,76-1,48$ & 249 & 149 & 50,5 & 0,56 & $0,31-1,00$ \\
\hline Sem rede geral & 72 & 15 & 4,3 & 0,63 & $0,32-1,23$ & 33 & 21 & 7,1 & 0,66 & $0,26-1,69$ \\
\hline Canalização interna & & & & & & & & & & \\
\hline $\operatorname{Sim}$ & 1.135 & 332 & 94,3 & 1,00 & - & 457 & 286 & 95,3 & 1,00 & - \\
\hline Não & 74 & 20 & 5,7 & 0,90 & $0,51-1,56$ & 21 & 14 & 4,7 & 1,20 & $0,44-3,34$ \\
\hline Distrito Sanitário de residêr & & & & & & & & & & \\
\hline$\|$ & 650 & 202 & 57,2 & 1,22 & $0,95-1,58$ & 257 & 163 & 54,2 & 1,00 & - \\
\hline IV & 561 & 151 & 42,8 & 1,00 & - & 222 & 138 & 45,8 & 0,95 & $0,64-1,40$ \\
\hline
\end{tabular}

(continua) 


\begin{tabular}{|c|c|c|c|c|c|c|c|c|c|c|}
\hline \multirow[t]{4}{*}{ Variáveis/Categorias } & \multicolumn{10}{|c|}{ Faixa etária } \\
\hline & \multicolumn{5}{|c|}{ 18-36 meses } & \multicolumn{5}{|c|}{5 anos } \\
\hline & \multirow[t]{2}{*}{ Total } & \multicolumn{2}{|c|}{$\begin{array}{c}\text { Casos } \\
\text { ceo-d } \geq 1\end{array}$} & \multirow[t]{2}{*}{ OR } & \multirow[t]{2}{*}{ IC95\% } & \multirow[t]{2}{*}{ Total } & \multicolumn{2}{|c|}{$\begin{array}{c}\text { Casos } \\
\text { ceo-d } \geq 1\end{array}$} & \multirow[t]{2}{*}{ OR } & \multirow[t]{2}{*}{ IC95\% } \\
\hline & & $\mathbf{n}$ & $\%$ & & & & $\mathrm{n}$ & $\%$ & & \\
\hline \multicolumn{11}{|c|}{ Tempo de moradia na área (anos) } \\
\hline Até 1 & 184 & 39 & 11,1 & 1,00 & - & 77 & 39 & 13,1 & 1,00 & - \\
\hline 2 & 373 & 77 & 21,9 & 0,97 & $0,61-1,53$ & 86 & 52 & 17,4 & 1,49 & $0,76-2,91$ \\
\hline 3 ou mais & 647 & 235 & 67,0 & 2,12 & $1,41-3,19$ & 311 & 207 & 69,5 & 1,94 & $1,14-3,31$ \\
\hline \multicolumn{11}{|l|}{ Faixa etária (meses) } \\
\hline Até 24 & 195 & 32 & 9,1 & 1,00 & - & & & & & \\
\hline $25-30$ & 157 & 28 & 7,9 & 1,11 & $0,61-2,00$ & & & & & \\
\hline $31-36$ & 859 & 293 & 83,0 & 2,64 & $1,73-4,04$ & & & & & \\
\hline \multicolumn{11}{|l|}{ Bloco estrutura familiar } \\
\hline \multicolumn{11}{|l|}{ Com quem a criança mora } \\
\hline Com os pais & 757 & 210 & 59,8 & 1,00 & - & 267 & 163 & 54,7 & 1,00 & - \\
\hline Mãe & 342 & 105 & 29,9 & 1,15 & $0,86-1,54$ & 150 & 93 & 31,2 & 1,04 & $0,68-1,60$ \\
\hline Pai/Outra situação & 110 & 36 & 10,3 & 1,27 & $0,81-1,99$ & 58 & 42 & 14,1 & 1,67 & $0,86-3,29$ \\
\hline \multicolumn{11}{|l|}{ Expediente em que a } \\
\hline \multicolumn{11}{|l|}{ mãe trabalha } \\
\hline Em horário integral & 230 & 52 & 15,2 & 1,00 & - & 87 & 53 & 18,1 & 0,82 & $0,49-1,38$ \\
\hline Meio expediente/ & 143 & 45 & 13,2 & 1,57 & $0,96-2,58$ & 70 & 39 & 13,3 & 0,66 & $0,38-1,16$ \\
\hline \multicolumn{11}{|l|}{ Alguns dias da semana } \\
\hline Não trabalha fora & 807 & 244 & 71,6 & 1,48 & $1,04-2,12$ & 307 & 201 & 68,6 & 1,00 & - \\
\hline \multicolumn{11}{|l|}{ Número de irmãos que } \\
\hline \multicolumn{11}{|l|}{ moram na casa } \\
\hline Nenhum & 436 & 107 & 30,8 & 1,00 & - & 101 & 50 & 17,1 & 1,00 & - \\
\hline 1 & 339 & 103 & 29,7 & 1,34 & $0,96-1,87$ & 155 & 95 & 32,6 & 1,61 & $0,94-2,77$ \\
\hline 2 & 239 & 67 & 19,3 & 1,20 & $0,83-1,74$ & 115 & 74 & 25,3 & 1,84 & $1,03-3,30$ \\
\hline 3 ou mais & 171 & 70 & 20,2 & 2,13 & $1,44-3,15$ & 96 & 73 & 25,0 & 3,24 & $1,69-6,25$ \\
\hline \multicolumn{11}{|l|}{ Ordem de nascimento } \\
\hline Não se aplica (filho único) & 283 & 67 & 19,1 & 1,00 & - & 70 & 33 & 11,0 & 1,00 & - \\
\hline Primeiro & 241 & 75 & 21,4 & 1,46 & $0,97-2,18$ & 138 & 91 & 30,4 & 2,17 & $1,16-4,08$ \\
\hline Intermediário & 127 & 44 & 12,6 & 1,71 & $1,05-2,77$ & 99 & 71 & 23,7 & 2,84 & $1,43-5,69$ \\
\hline Último & 543 & 164 & 46,9 & 1,40 & $0,99-1,97$ & 169 & 104 & 34,9 & 1,79 & $0,98-3,27$ \\
\hline
\end{tabular}

IC95\%: intervalo de 95\% de confiança; OR: odds ratio.

$(\mathrm{OR}=2,05$; IC95\%: 1,18-3,57) e procurar serviços de saúde bucal para tratamento $(\mathrm{OR}=5,74$; IC95\%: 2,48-13,28) e para o atendimento de emergência (OR = 3,60; IC95\%: 1,10-1,64). Esses fatores foram associados positivamente com a experiência de cárie nesta idade (Tabela 4).

\section{Discussão}

Mesmo tratando-se de uma população relativamente homogênea em relação às precárias condições socioeconômicas, algumas exposições apresentaram distribuições heterogêneas, sendo capazes de discriminar a ocorrência da doença. Residir em moradias com maior número de pes- soas e a baixa escolaridade do cuidador da criança (predominantemente materna), variáveis proxi de condições de vida desfavoráveis, foram preditores de cárie dentária neste trabalho.

Tais resultados estão de acordo com estudos prévios que investigaram a influência dos fatores socioambientais na saúde bucal da população infantil 6,8,9,22,23,24 e sugerem que os fatores de risco contextuais na produção da cárie, nessa população, não estão sendo enfrentados.

Como demonstrado em outros estudos $5,15,17,22,25$, para as crianças com idades entre 1836 meses, o risco de cárie aumentou com a idade. Uma das possíveis explicações esse aumento está relacionada ao acúmulo de riscos sociobiológicos atuando continuamente com o avançar da 
Análise univariada da associação entre a experiência cárie dentária e variáveis relacionadas aos cuidados com a saúde bucal e ao uso de serviços de educação e saúde bucal, nas faixas etárias de 18-36 meses e 5 anos de idade. Recife, Pernambuco, Brasil, 2006.

\begin{tabular}{|c|c|c|c|c|c|c|c|c|c|c|}
\hline \multirow[t]{4}{*}{ Variáveis/Categorias } & \multicolumn{10}{|c|}{ Faixa etária } \\
\hline & \multicolumn{5}{|c|}{$18-36$ meses } & \multicolumn{5}{|c|}{5 anos } \\
\hline & \multirow[t]{2}{*}{ Total } & \multicolumn{2}{|c|}{$\begin{array}{c}\text { Casos } \\
\text { ceo- } d \geq 1\end{array}$} & \multirow[t]{2}{*}{ OR } & \multirow[t]{2}{*}{ IC95\% } & \multirow[t]{2}{*}{ Total } & \multicolumn{2}{|c|}{$\begin{array}{c}\text { Casos } \\
\text { ceo- } d \geq 1\end{array}$} & \multirow[t]{2}{*}{ OR } & \multirow[t]{2}{*}{ IC95\% } \\
\hline & & $n$ & $\%$ & & & & $n$ & $\%$ & & \\
\hline \multicolumn{11}{|l|}{ Bloco cuidados com a saúde bucal } \\
\hline \multicolumn{11}{|l|}{ Cuidador diário da criança } \\
\hline Mãe & 823 & 246 & 69,9 & 1,00 & - & 352 & 219 & 72,8 & 1,00 & - \\
\hline Creche & 69 & 27 & 7,7 & 1,51 & $0,88-2,57$ & 9 & 4 & 1,3 & 0,49 & $0,11-2,13$ \\
\hline Pai/Avós/Outros & 317 & 79 & 22,4 & 0,78 & $0,57-1,06$ & 118 & 78 & 25,9 & 1,18 & $0,75-1,88$ \\
\hline \multicolumn{11}{|l|}{ Escolaridade do cuidador } \\
\hline \multicolumn{11}{|l|}{ (anos de estudo) } \\
\hline$<8$ & 638 & 188 & 56,1 & 1,42 & $0,97-2,08$ & 270 & 198 & 67,1 & 4,26 & $2,44-7,46$ \\
\hline $8-10$ & 307 & 99 & 29,6 & 1,62 & $1,06-2,46$ & 119 & 66 & 22,4 & 1,93 & $1,04-3,59$ \\
\hline 11 ou mais & 211 & 48 & 14,3 & 1,00 & - & 79 & 31 & 10,5 & 1,00 & - \\
\hline \multicolumn{11}{|l|}{ Ingestão de doces entre } \\
\hline \multicolumn{11}{|l|}{ as refeições } \\
\hline Não & 135 & 29 & 8,2 & 1,00 & - & 46 & 19 & 6,3 & 1,00 & - \\
\hline Às vezes & 620 & 140 & 39,7 & 1,07 & $0,66-1,72$ & 193 & 101 & 33,6 & 1,56 & $0,78-3,15$ \\
\hline Diariamente & 454 & 184 & 52,1 & 2,49 & $1,55-4,02$ & 240 & 181 & 60,1 & 4,36 & $2,16-8,86$ \\
\hline \multicolumn{11}{|l|}{ Uso de leite materno presente } \\
\hline Sim & 171 & 54 & 15,3 & 1,15 & $0,80-1,65$ & 9 & 3 & 1,0 & 0,29 & $0,06-1,31$ \\
\hline Não & 1.038 & 298 & 84,7 & 1,00 & - & 470 & 298 & 99,0 & 1,00 & - \\
\hline \multicolumn{11}{|l|}{ Uso de mamadeira noturna } \\
\hline Sim & 593 & 142 & 40,3 & 0,60 & $0,47-0,78$ & 99 & 59 & 19,7 & 0,84 & $0,52-1,36$ \\
\hline Não & 613 & 210 & 59,7 & 1,00 & - & 379 & 241 & 80,3 & 1,00 & - \\
\hline Periodicidade da escovação & & & & & & & & & & \\
\hline Não realiza/Às vezes & 157 & 47 & 13,4 & 1,04 & $0,71-1,53$ & 64 & 47 & 15,7 & 1,76 & $0,94-3,30$ \\
\hline Diariamente & 1.049 & 305 & 86,6 & 1,00 & - & 412 & 252 & 84,3 & 1,00 & - \\
\hline Frequência diária de escovação & & & & & & & & & & \\
\hline Não realiza/Às vezes & 157 & 47 & 13,4 & 1,06 & $0,70-1,59$ & 64 & 47 & 15,7 & 2,02 & $1,03-3,89$ \\
\hline $1 \mathrm{vez}$ & 151 & 42 & 11,9 & 0,95 & $0,62-1,45$ & 57 & 38 & 12,7 & 1,46 & $0,75-2,87$ \\
\hline 2 vezes & 374 & 112 & 31,8 & 1,06 & $0,78-1,43$ & 175 & 110 & 36,8 & 1,24 & $0,79-1,94$ \\
\hline 3 vezes ou mais & 524 & 151 & 42,9 & 1,00 & - & 180 & 104 & 34,8 & 1,00 & - \\
\hline Uso de pasta dental & & & & & & & & & & \\
\hline Sim & 1.131 & 338 & 95,8 & 1,00 & - & 455 & 283 & 94,3 & 1,00 & - \\
\hline Não/Não se aplica & 78 & 15 & 4,2 & 0,53 & $0,30-1,02$ & 23 & 17 & 5,7 & 1,72 & $0,62-4,99$ \\
\hline Quem realiza a escovação & & & & & & & & & & \\
\hline Adulto & 853 & 248 & 70,4 & 1,00 & - & 135 & 77 & 25,7 & 1,00 & - \\
\hline Criança com ajuda do adulto & 188 & 57 & 16,1 & 1,06 & $0,74-1,52$ & 103 & 62 & 20,7 & 1,14 & $0,65-1,98$ \\
\hline Criança & 124 & 39 & 11,0 & 1,12 & $0,73-1,71$ & 227 & 150 & 49,9 & 1,47 & $0,92-2,33$ \\
\hline Não realiza limpeza & 45 & 9 & 2,5 & 0,61 & $0,27-1,34$ & 12 & 11 & 3,7 & 8,29 & $1,06-176,49$ \\
\hline Bloco uso de serviços de & & & & & & & & & & \\
\hline educação e saúde bucal & & & & & & & & & & \\
\hline Criança frequenta creche & & & & & & & & & & \\
\hline ou pré-escola & & & & & & & & & & \\
\hline Sim & 568 & 184 & 52,4 & 1,00 & - & 396 & 252 & 83,7 & 1,00 & - \\
\hline Não & 641 & 167 & 47,6 & 0,74 & $0,57-0,95$ & 83 & 49 & 16,3 & 0,82 & $0,49-1,37$ \\
\hline
\end{tabular}

(continua) 


\begin{tabular}{|c|c|c|c|c|c|c|c|c|c|c|}
\hline \multirow[t]{5}{*}{ Variáveis/Categorias } & \multicolumn{10}{|c|}{ Faixa etária } \\
\hline & \multicolumn{5}{|c|}{ 18-36 meses } & \multicolumn{5}{|c|}{5 anos } \\
\hline & \multirow[t]{3}{*}{ Total } & \multirow{2}{*}{\multicolumn{2}{|c|}{$\begin{array}{c}\text { Casos } \\
\text { ceo-d } \geq 1\end{array}$}} & \multirow[t]{3}{*}{ OR } & \multirow[t]{3}{*}{ IC95\% } & \multirow[t]{3}{*}{ Total } & \multirow{2}{*}{\multicolumn{2}{|c|}{$\begin{array}{c}\text { Casos } \\
\text { ceo-d } \geq 1\end{array}$}} & \multirow[t]{3}{*}{ OR } & \multirow[t]{3}{*}{ IC95\% } \\
\hline & & & & & & & & & & \\
\hline & & $\mathrm{n}$ & $\%$ & & & & $\mathrm{n}$ & $\%$ & & \\
\hline \multicolumn{11}{|c|}{ Tipo de creche ou pré-escola } \\
\hline Privada & 335 & 92 & 26,5 & 1,00 & - & 158 & 81 & 27,0 & 1,00 & - \\
\hline Pública & 200 & 77 & 22,1 & 1,65 & $1,12-2,44$ & 213 & 152 & 50,7 & 2,37 & $1,51-3,73$ \\
\hline Filantrópica & 26 & 12 & 3,4 & 2,26 & $0,94-5,43$ & 23 & 18 & 6,0 & 3,42 & $1,12-11,13$ \\
\hline Não frequenta & 641 & 167 & 48,0 & 0,93 & $0,68-1,27$ & 83 & 49 & 16,3 & 1,37 & $0,77-2,43$ \\
\hline \multicolumn{11}{|c|}{ Procura pelas ações de } \\
\hline \multicolumn{11}{|c|}{ saúde bucal oferecidas na USF } \\
\hline $\operatorname{Sim}$ & 501 & 153 & 45,3 & 1,00 & - & 270 & 183 & 62,7 & 1,00 & - \\
\hline Não & 670 & 185 & 54,7 & 0,87 & $0,67-1,13$ & 198 & 109 & 37,3 & 0,58 & $0,39-0,87$ \\
\hline \multicolumn{11}{|l|}{ Razão da procura } \\
\hline \multicolumn{11}{|c|}{ Educativa/Orientação } \\
\hline Sim & 266 & 68 & 20,1 & 1,00 & - & 126 & 81 & 27,7 & 1,00 & - \\
\hline Não & 905 & 270 & 79,9 & 1,24 & $0,90-1,71$ & 342 & 211 & 72,3 & 0,89 & $0,57-1,40$ \\
\hline \multicolumn{11}{|l|}{ Prevenção } \\
\hline $\operatorname{Sim}$ & 401 & 118 & 34,9 & 1,00 & - & 207 & 133 & 45,5 & 1,00 & - \\
\hline Não & 770 & 220 & 65,1 & 0,96 & $0,73-1,26$ & 261 & 159 & 54,5 & 0,87 & $0,58-1,29$ \\
\hline \multicolumn{11}{|l|}{ Tratamento } \\
\hline Sim & 72 & 34 & 10,1 & 2,34 & $1,41-3,89$ & 94 & 81 & 27,7 & 4,81 & $2,50-9,42$ \\
\hline Não & 1099 & 304 & 89,9 & 1,00 & - & 374 & 211 & 72,3 & 1,00 & - \\
\hline \multicolumn{11}{|l|}{ Emergência } \\
\hline Sim & 43 & 18 & 5,3 & 1,82 & $0,94-3,51$ & 41 & 36 & 12,3 & 4,81 & $1,76-14,24$ \\
\hline Não & 1128 & 320 & 94,7 & 1,00 & - & 427 & 256 & 87,7 & 1,00 & - \\
\hline \multicolumn{11}{|l|}{ Utiliza outros serviços } \\
\hline \multicolumn{11}{|l|}{ de saúde bucal } \\
\hline Sim & 218 & 72 & 21,2 & 1,26 & $0,91-1,75$ & 142 & 96 & 32,4 & 1,33 & $0,86-2,05$ \\
\hline Não & 950 & 267 & 78,8 & 1,00 & - & 327 & 200 & 67,6 & 1,00 & - \\
\hline
\end{tabular}

IC95\%: intervalo de 95\% de confiança; OR: odds ratio; USF: unidade de saúde da família.

idade 24. Peres et al. 24 acrescentam que os fenômenos relacionados à cárie infantil são fundamentalmente mais tardios, observando ser o agravo um evento basicamente social, nutricional e dietético. Nesse aspecto, estudo como o de Holm 26 confirma nosso achado, tendo sido observado que as condições de desvantagens sociais e materiais às quais os indivíduos estão expostos podem se expressar em uma resistência mais reduzida à cárie, por comprometer a capacidade em prover o autocuidado, entre outros fatores. Essas condições influenciam no risco de uma criança ser acometida pela cárie precocemente, a partir da erupção dos primeiros dentes, além de aumentar a probabilidade de desenvolver cárie subsequente nas duas dentições 11. Ademais, há ainda as questões culturais e de desvalorização da dentição decídua por parte dos responsáveis pelas crianças $6,7,8$.
Residir em domicílios abastecidos por poço ou nascente constituiu um fator de proteção contra a cárie entre as crianças nessa faixa etária, em comparação com as que residiam em domicílios abastecidos pela rede geral. Dificuldade no acesso a abastecimento de água regular e de qualidade tem sido fator relacionado a inúmeros agravos à saúde na infância, como doença diarréica 27 . A exemplo do que ocorre em várias cidades da Região Nordeste, a oferta irregular de água tratada pela rede pública de abastecimento no Recife tem constituído um problema que atinge a maior parte do município; para compensar essa irregularidade, parte da população utiliza água proveniente de outras fontes, como, por exemplo, poços. Possivelmente, a melhoria da oferta de água proporcionada por essa outra fonte de abastecimento tenha induzido melhores práticas de higiene bucal dessas crianças, reduzindo, 
Modelo final para os fatores associados à experiência de cárie dentária nas crianças de 18-36 meses de idade: análise multivariada. Recife, Pernambuco, Brasil, 2006.

\begin{tabular}{|c|c|c|c|c|}
\hline Variáveis/Categorias & $\begin{array}{l}\text { ORajustada } 1 \\
\text { OR (IC95\%) }\end{array}$ & $\begin{array}{l}\text { Valor de } p \\
\text { ajustada } 1\end{array}$ & $\begin{array}{l}\text { ORajustada } 2 \\
\text { OR (IC95\%) }\end{array}$ & $\begin{array}{c}\text { Valor de } p \\
\text { ajustada } 2\end{array}$ \\
\hline Faixa etária (meses) & & & & 0,009 \\
\hline Até 24 & 1,00 & - & 1,00 & - \\
\hline $25-30$ & $1,18(0,63-2,23)$ & 0,610 & $1,53(0,78-3,01)$ & 0,218 \\
\hline $31-36$ & $1,97(1,18-3,28)$ & 0,010 & $2,29(1,32-3,97)$ & 0,003 \\
\hline \multicolumn{5}{|l|}{ Número de pessoas por quarto de dormir } \\
\hline Até 3 & 1,00 & - & 1,00 & - \\
\hline Mais de 3 & $1,71(1,30-2,25)$ & $<0,001$ & $1,68(1,23-2,29)$ & 0,001 \\
\hline Origem da água do domicílio & & & & 0,075 \\
\hline Rede geral & 1,00 & - & 1,00 & - \\
\hline Poço/Nascente & $0,42(0,18-0,96)$ & 0,039 & $0,36(0,15-0,88)$ & 0,024 \\
\hline Outra situação & $1,51(0,58-3,90)$ & 0,398 & $0,80(0,27-2,38)$ & 0,693 \\
\hline Tempo de moradia na área (anos) & & & & 0,056 \\
\hline Até 1 & 1,00 & - & 1,00 & - \\
\hline 2 & $0,81(0,49-1,33)$ & 0,399 & $0,79(0,47-1,34)$ & 0,381 \\
\hline 3 ou mais & $1,43(0,89-2,32)$ & 0,142 & $1,27(0,77-2,11)$ & 0,355 \\
\hline Número de irmãos que moram na casa & & & & 0,039 \\
\hline Nenhum & 1,00 & - & 1,00 & - \\
\hline 1 & $1,32(0,95-1,83)$ & 0,101 & $1,08(0,75-1,55)$ & 0,697 \\
\hline 2 & $1,17(0,81-1,70)$ & 0,399 & $0,88(0,59-1,33)$ & 0,554 \\
\hline 3 ou mais & $2,13(1,44-3,14)$ & $<0,001$ & $1,71(1,10-2,66)$ & 0,018 \\
\hline Cuidador diário da criança & & & * & * \\
\hline Mãe & 1,00 & - & & \\
\hline Creche & $2,07(1,05-4,05)$ & 0,035 & & \\
\hline Pai/Avós/Outros & $0,73(0,53-1,01)$ & 0,055 & & \\
\hline Escolaridade do cuidador (anos de estudo) & & & * & * \\
\hline$<8$ & $1,49(1,01-2,18)$ & 0,041 & & \\
\hline $8-10$ & $1,63(1,07-2,48)$ & 0,023 & & \\
\hline 11 ou mais & 1,00 & - & & \\
\hline Ingestão de doces entre as refeições & & & & 0,000 \\
\hline Não & 1,00 & - & 1,00 & - \\
\hline Às vezes & $1,15(0,70-1,89)$ & 0,574 & $1,11(0,66-1,87)$ & 0,698 \\
\hline Diariamente & $2,84(1,73-4,65)$ & $<0,001$ & $2,81(1,66-4,73)$ & $<0,001$ \\
\hline \multicolumn{5}{|l|}{ Uso de mamadeira noturna presente } \\
\hline $\operatorname{Sim}$ & $0,64(0,49-0,84)$ & 0,001 & $0,68(0,51-0,90)$ & 0,007 \\
\hline Não & 1,00 & - & 1,00 & - \\
\hline Tipo de creche ou pré-escola & & & * & * \\
\hline Privada & 1,00 & - & & \\
\hline Pública & $1,76(1,19-2,60)$ & 0,005 & & \\
\hline Filantrópica & $1,93(0,81-4,63)$ & 0,140 & & \\
\hline Não frequenta & $1,24(0,90-1,71)$ & 0,197 & & \\
\hline \multicolumn{5}{|l|}{ Razão da procura } \\
\hline \multicolumn{5}{|l|}{ Tratamento } \\
\hline Sim & $2,15(1,30-3,56)$ & 0,003 & $2,05(1,20-3,50)$ & 0,009 \\
\hline Não & 1,00 & - & 1,00 & - \\
\hline
\end{tabular}

Nota: ajustada 1: ajustada dentro do bloco e incluída na análise múltipla final ( $p<0,10)$; ajustada 2: ajustada no modelo final. IC95\%: intervalo de 95\% de confiança; OR: odds ratio.

* Retirada da análise múltipla final. 
Tabela 4

Modelo final para os fatores associados à experiência de cárie dentária nas crianças de cinco anos de idade: análise multivariada. Recife, Pernambuco, Brasil, 2006.

\begin{tabular}{|c|c|c|c|c|}
\hline Variáveis/Categorias & $\begin{array}{l}\text { ORajustada } 1 \\
\text { OR (IC95\%) }\end{array}$ & $\begin{array}{l}\text { Valor de } p \\
\text { ajustada } 1\end{array}$ & $\begin{array}{l}\text { ORajustada } 2 \\
\text { OR (IC95\%) }\end{array}$ & $\begin{array}{c}\text { Valor de } p \\
\text { ajustada } 1\end{array}$ \\
\hline Escolaridade materna (anos de estudo) & & & * & * \\
\hline$<8$ & $3,55(2,14-5,89)$ & $<0,001$ & & \\
\hline $8-10$ & $2,08(1,18-3,68)$ & 0,012 & & \\
\hline 11 ou mais & 1,00 & - & & \\
\hline Moradores por domicílio & & & & 0,002 \\
\hline Até 3 & 1,00 & - & 1,00 & - \\
\hline $4-5$ & $2,18(1,28-3,73)$ & 0,004 & $1,81(0,96-3,44)$ & 0,069 \\
\hline 6 ou mais & $3,46(1,91-6,25)$ & $<0,001$ & $3,46(1,70-7,00)$ & 0,001 \\
\hline Com quem a criança mora & 1,00 & - & * & * \\
\hline \multicolumn{5}{|l|}{ Com os pais } \\
\hline Mãe & $1,12(0,73-1,72)$ & 0,613 & & \\
\hline Pai/Outra situação & $3,17(1,44-6,96)$ & 0,004 & & \\
\hline Número de irmãos que moram na casa & & & * & * \\
\hline Nenhum & 1,00 & - & & \\
\hline 1 & $1,56(0,91-2,66)$ & 0,104 & & \\
\hline 2 & $1,98(1,11-3,50)$ & 0,020 & & \\
\hline 3 ou mais & $3,46(1,81-6,59)$ & $<0,001$ & & \\
\hline Escolaridade do cuidador (anos de estudo) & & & & 0,001 \\
\hline$<8$ & $3,87(2,25-6,65)$ & $<0,001$ & $2,95(1,53-5,67)$ & 0,001 \\
\hline $8-10$ & $2,02(1,10-3,70)$ & 0,023 & $1,33(0,65-2,71)$ & 0,432 \\
\hline 11 ou mais & 1,00 & - & 1,00 & - \\
\hline Ingestão de doces entre as refeições & & & & 0,000 \\
\hline Não & 1,00 & - & 1,00 & - \\
\hline Às vezes & $1,50(0,76-2,99)$ & 0,243 & $1,18(0,53-2,67)$ & 0,683 \\
\hline Diariamente & $3,85(1,94-7,65)$ & $<0,001$ & $3,85(1,71-8,63)$ & 0,001 \\
\hline Uso de leite materno atualmente & & & * & * \\
\hline $\operatorname{Sim}$ & $0,25(0,06-1,04)$ & 0,056 & & \\
\hline Não & 1,00 & - & & \\
\hline Tipo de creche ou pré-escola & & & & 0,025 \\
\hline Privada & 1,00 & - & 1,00 & - \\
\hline Pública & $2,44(1,54-3,87)$ & $<0,001$ & $2,05(1,18-3,57)$ & 0,011 \\
\hline Filantrópica & $4,03(1,26-12,92)$ & 0,019 & $2,87(0,73-11,26)$ & 0,131 \\
\hline Não freqüenta & $1,43(0,79-2,57)$ & 0,235 & $1,00(0,51-1,96)$ & 0,993 \\
\hline \multicolumn{5}{|l|}{ Razão da procura } \\
\hline \multicolumn{5}{|l|}{ Tratamento } \\
\hline Sim & $4,87(2,38-9,99)$ & $<0,001$ & $5,74(2,48-13,28)$ & $<0,001$ \\
\hline Não & 1,00 & - & 1,00 & - \\
\hline \multicolumn{5}{|l|}{ Emergência } \\
\hline Sim & $2,87(1,02-8,04)$ & 0,045 & $3,60(1,10-11,64)$ & 0,033 \\
\hline Não & 1,00 & - & 1,00 & - \\
\hline Utiliza outros serviços de saúde bucal & & & * & * \\
\hline $\operatorname{Sim}$ & $1,49(0,95-2,34)$ & 0,086 & & \\
\hline Não & 1,00 & - & & \\
\hline
\end{tabular}

Nota: ajustada 1: ajustada dentro do bloco e incluída na análise múltipla final $(p<0,10)$; ajustada 2: ajustada no modelo final. IC95\%: intervalo de 95\% de confiança; OR: odds ratio.

* Retirada da análise múltipla final. 
assim, o risco de cárie. No entanto, estudos anteriores que investigaram a relação entre o acesso à água no domicílio e a ocorrência de cárie entre crianças de 33-48 meses 15 e de seis anos 24 não constataram tal associação.

Sobre os aspectos relacionados à estrutura familiar, verificou-se que apenas a existência de três ou mais irmãos morando na mesma casa foi preditora de cárie nessa faixa etária, achado que é consistente com estudos que indicaram menor risco de cárie entre as crianças que não tinham irmãos ou os tinham em menor número 22,28. Em São Paulo 15, verificou-se que as categorias de crianças com maiores chances de ter cárie foram aquelas que tinham mais de quatro irmãos. No Canadá 29 , um estudo em crianças de três anos mostrou que o tamanho da família (cinco ou mais pessoas) foi uma variável que apresentou uma forte associação com a cárie.

De acordo com nosso estudo, pode-se supor que a existência de arranjos familiares numerosos representa obstáculos ao cuidado da saúde dentária das crianças a partir dos primeiros anos de vida. Reforça-se, assim, a importância de maior ênfase nas estratégias promocionais de saúde, com aplicação de medidas de caráter intersetorial nas áreas estudadas, uma vez que, concordando com Vasconcelos 30 , nas classes populares, as famílias vivem em situações especiais de risco, o que as torna incapazes de efetivamente articular os cuidados entre seus membros.

Nos resultados relacionados aos cuidados com a saúde bucal, o consumo diário de doces, nas duas faixas etárias, apresentou-se como um fator associado ao desfecho observado. Esse resultado reforça achados de estudos sobre o padrão de ingestão de sacarose, particularmente entre as refeições e/ou quando há um prolongado contato de carboidratos fermentáveis sobre os dentes, e o risco de cárie 31,32,33. Em crianças muito jovens, o alto consumo de açúcar pode ocasionar padrões de acometimentos de cárie mais graves, que interferem na sua qualidade de vida 32,33 .

Condicionantes socioeconômicos e nutricionais e fatores como imaturidade do órgão dental, hipoplasia dentária por desnutrição, frequência e qualidade da escovação dentária, baixa exposição ao flúor, entre outros, demonstram que o açúcar se associa à cárie em pré-escolares $5,22,24,34,35$, conclusão encontrada também no presente estudo. Todavia, alguns trabalhos mostraram resultados distintos 15,36, ou informam uma fraca correlação entre o consumo de sacarose e a cárie, especialmente em populações expostas a programas educativos, com uso de guias alimentares e dos fluoretos 35,37. As discordâncias entre os estudos devem-se a possíveis vieses de informação e a possíveis variáveis de confundimento, tais como acesso ao flúor e ao padrão nutricional, que precisam ser levados em consideração nas análises 37 . Em nosso estudo, em virtude do expressivo percentual de respondentes com baixa escolaridade, aspectos relacionados ao entendimento das perguntas podem ter conduzido a esse viés de informação.

Entretanto, concordamos com Aquino \& Philippi 38 , segundo os quais uma menor renda familiar é associada ao consumo maior de alimentos de baixo valor nutritivo pelas crianças, como o açúcar. Essa evidência parece corroborar as fortes associações encontradas entre o consumo de doces e a cárie das crianças examinadas, as quais vivem em áreas sem a proteção da fluoretação das águas de abastecimento público. Por outro lado, a tendência de crescimento do consumo de açúcar no mundo e o elevado padrão nacional de consumo do produto, juntamente com o processo de transição alimentar, vêm exigindo a adoção de políticas nutricionais mais integrais 38,39 , a fim de provocar modificação na prevalência desse fator de risco, envolvido não só no maior incremento da cárie, como também em diversos agravos infantis relacionados ao padrão alimentar 39 .

Quanto ao uso da mamadeira noturna, em contraposição às pesquisas que relacionaram o seu uso à cárie precoce $4,5,40$, em nosso estudo esse hábito constituiu fator de proteção para a cárie nas crianças entre 18-36 meses, resultados consistentes com os de outros autores 15,36. Esse fato instiga reflexões que não foram consideradas neste estudo, podendo ter enviesado os resultados, tais como: tipo de alimento, adoção de açúcar, frequência de ingestão, tempo em que a mamadeira fica na boca, interposição da higiene dentária após o uso da mamadeira noturna, entre outros 5. Por outro lado, nas áreas estudadas, merece ser investigado o impacto de possíveis ações promocionais e educativas, implementadas pela ESF, sobre os efeitos nocivos desse hábito alimentar na saúde dentária das crianças.

Além disso, as divergências de resultados sobre o uso da mamadeira noturna devem ser analisadas considerando-se a complexidade do processo saúde-doença, que exige uma abordagem hierárquica entre fatores de risco sociais, comportamentais e biológicos, segundo o contexto social onde as doenças são produzidas 2,6,7,8,9. Nesse sentido, o efeito do uso da mamadeira noturna com leite ou com outras substâncias (independente de ser ou não açucarada) sobre a ocorrência de cárie, nos primeiros anos de vida, pode apresentar variações. Tinanoff \& O’Sullivan 41 salientam que, da mesma maneira que a cárie 
está frequentemente associada ao uso de mamadeira, $69 \%$ de crianças livres de cárie também tomam mamadeira na cama.

Na população de cinco anos, ratificando outros estudos 5,15,22,29, além da variável residir em domicílios com um maior número de moradores, a baixa escolaridade do cuidador, predominantemente representado pelas mães, constituiu um risco para a cárie. Crianças cujo cuidador tinha até oito anos de estudo tiveram chance quase três vezes maior de apresentar cárie em relação às crianças que tinham cuidadores com 11 anos $\mathrm{e}$ mais de estudo. Resultados semelhantes também foram observados em São Paulo 15 no Programa Saúde da Família, porém diferem dos de Brandão et al. ${ }^{36}$. Esse achado destaca a necessidade de efetivas políticas educacionais locais para a melhoria do nível educacional dos pais das famílias das áreas investigadas.

A influência da pré-escola na cárie infantil apresentou, neste estudo, associações com a experiência de cárie apenas aos cinco anos. Crianças que frequentavam pré-escola pública apresentaram duas vezes mais chances de ter cárie, quando comparadas àquelas que não frequentavam pré-escola. Esses resultados estão de acordo com os de outros estudos, que mostraram que crianças de pré-escolas públicas apresentam uma probabilidade mais elevada de ter cárie, quando comparadas às de pré-escolas privadas 23,25 .

A pré-escola é concebida como um espaço promotor do desenvolvimento integral da criança, na sua socialização e construção de comportamentos favoráveis à saúde. Entretanto, em contextos sociais heterogêneos como no Brasil, a variável tipo de escola, se constitui uma proxi das condições socioeconômicas das crianças de escolas públicas, que majoritariamente pertencem aos extratos sociais mais pobres. Além do que, dificuldades na alocação de investimentos públicos para melhoria da infraestrutura e da qualidade do ensino das pré-escolas públicas, com a inclusão de propostas promotoras da saúde 35 , são constantemente relatadas. Sendo assim, as características socioculturais das famílias e aspectos contextuais das áreas estudadas, como a localização geográfica da escola em áreas de risco social do Recife, podem ter influenciado nessa predição

Aspectos pertinentes à utilização dos serviços de saúde bucal nas USF e o resultado encontrado, segundo o qual a razão da procura é mais para tratamento e/ou emergência em consequência do ataque de cárie, assim como em outros trabalhos 25,34,42, foram fatores explicativos que apresentaram fortes associações com o desfecho estudado nessa população. Tais resultados podem estar indicando dificuldades tanto no processo de substituição de práticas curativas por práticas pautadas no referencial do modelo de promoção à saúde, quanto na realização de ações integradas à saúde materno-infantil e familiar, organizadas para a linha de cuidado préescolar 2,11,16, propostas para as áreas estudadas. Ademais, vale salientar que as políticas públicas de saúde bucal no Brasil e no Recife são recentes, tendo, até então, se mostrado pouco efetivas para adequar os serviços odontológicos às necessidades acumuladas de tratamento de cárie da maioria da população. Isso significa um nó crítico para a ampliação do acesso e da qualificação da atenção à saúde no SUS 11,12,14.

A esse respeito, a alta prevalência de dentes cariados não tratados, que predominou nos valores do índice ceo-d das crianças examinadas (1836 meses: 93,8\%; 5 anos: 81,7,\%)17, pode explicar, em parte, uma maior procura às USF para tratamento ou alívio da dor. Esse achado está em conformidade com os resultados do último levantamento nacional, que identificou uma enorme demanda reprimida por tratamento odontológico nessas faixas etárias, principalmente no Norte e Nordeste do país 10. Narvai et al. 2 comentam que, a despeito do pouco impacto dos serviços de saúde nos níveis de cárie, no Brasil valoriza-se a influência de diversos programas de saúde bucal municipais, os quais atuam sob o paradigma da promoção à saúde, no decréscimo da cárie.

Nesse sentido, para qualificar a atenção direcionada aos pré-escolares, historicamente excluídos das políticas de saúde bucal 2,14,16, são enfatizadas as recomendações dos estudos que comprovam o efeito de práticas e serviços de promoção à saúde bucal na qualidade de saúde bucal infantil, a partir do nascimento. Realizadas em diversas cidades brasileiras, essas práticas são uma tentativa de enfrentar os problemas bucais de maneira precoce, principalmente a cárie dentária 44 .

No entanto, os resultados alcançados neste estudo devem ser analisados com cautela, tendo em vista as limitações inerentes ao desenho de estudo transversal adotado, do qual os dados para este trabalho foram retirados. Assim, foram utilizadas estratégias metodológicas para assegurar a validade do estudo, a confiabilidade e reprodutibilidade dos dados. O possível viés de seleção, decorrente dos percentuais de perdas, identificados como consequências de falhas no processo de cadastramento das famílias nas USF, foi minimizado na análise mediante a ponderação das taxas de prevalência 17. Além disso, pode-se supor que os resultados obtidos não se encontram enviesados, dado que todas as crianças eram oriundas de unidades do mesmo porte 
(USF) e possuíam relativa homogeneidade do ponto de vista socioeconômico. Deve-se tomar cuidado, também, com as generalizações dos resultados, uma vez que a população estudada é originária de áreas urbanas pobres e assistida pelas ações da ESF local. Estudos dirigidos a populações semelhantes são necessários para confirmar esses achados.

Em síntese, em áreas com precárias condições socioeconômicas foi possível identificar distintos fatores que contribuíram para o agravo em ambas as faixas etárias pesquisadas. De uma maneira geral, as informações produzidas neste estudo expressam a acumulação de uma série de problemas sociais não enfrentados, evidenciando iniquidades em saúde em áreas intraurbanas do Recife. Para uma modificação intencional no quadro epidemiológico apresentado pelos préescolares estudados, são indicadas ações integradas de saúde comuns a outros agravos infantis em diversos níveis do sistema de saúde, que combinem políticas sociais e de saúde pública com as formuladas para a operacionalização da Atenção Primária à Saúde do Recife, focadas na equidade.

\section{Resumo}

Para investigar fatores associados à cárie dentária na dentição decídua, realizou-se estudo de caso-controle aninhado a inquérito de prevalência em 1.690 pré-escolares de 18-36 meses e cinco anos de idade, assistidos pela Estratégia Saúde da Família no Recife, Pernambuco, Brasil, em 2006. Os casos foram crianças com ceo- $d \geq 1$ e os controles, com ceo- $d=0$. As variáveis independentes foram analisadas em blocos sociodemográfico e ambiental, estrutura familiar, cuidados com a saúde bucal e uso de serviços. Estimou-se OR brutas e IC95\%. Variáveis associadas ao desfecho com $p \leq 0,20$ em análises univariadas foram incluídas no modelo de regressão logística multivariado, com critério de permanência no modelo final $p \leq 0,10$. Maior número de moradores no domicílio, falta de abastecimento de água, tempo de moradia, escolaridade do cuidador, uso de pré-escola pública, procura por serviço de saúde bucal e padrão de consumo de açúcar foram fatores associados. As variáveis relacionadas ao contexto social foram os principais preditores de cárie, apontando para a necessidade de ações integradas de saúde comuns a outros agravos infantis.

Cárie Dentária; Pré-Escolar; Condições Sociais; Serviços de Saúde Bucal

\section{Colaboradores}

M. M. D. C. Melo participou do planejamento e execução do projeto, análise e interpretação dos dados, redação do manuscrito e aprovação da versão final deste. W. V. Souza contribuiu na orientação do estudo, análise e interpretação dos dados, e aprovou a versão final do manuscrito. M. L. C. Lima participou do planejamento do estudo, interpretação dos dados, revisão e aprovação final do manuscrito. C. Braga colaborou na interpretação dos dados, revisão crítica do manuscrito, e aprovou a versão final deste.

\section{Agradecimentos}

Às crianças e aos seus responsáveis por autorizarem os exames; à Secretaria de Saúde do Recife, por meio da Coordenadora de Saúde Bucal, Dra. Nilcema Figueiredo, e das Gerências dos Distritos Sanitários II e IV, com a participação das suas equipes de saúde bucal. 


\section{Referências}

1. McGrath C, Broder H, Wilson-Genderson M. Assessing the impact of oral health on the life quality of children: implications for research and practice. Community Dent Oral Epidemiol 2004; 32:81-5.

2. Narvai PC, Frazão P, Roncalli AG, Antunes JLF. Cárie dentária no Brasil: declínio, polarização, iniqüidade e exclusão social. Rev Panam Salud Pública 2006; 19:385-93.

3. Marthaler TM. Change in dental caries in Europe 1953-2003. Caries Res 2004; 38:173-81.

4. Hallett KB, O'Rourke PK. Pattern and severity of early childhood caries. Community Dent Oral Epidemiol 2006; 34:25-35.

5. Dini EL, Holt RD, Bedi R. Caries and its association with infant feeding and oral health-related behaviours in 3-4-year-old Brazilian children. Community Dent Oral Epidemiol 2000; 28:241-8.

6. Mello RC, Antunes JLF, Waldman EA. Prevalência de cárie não tratada na dentição decídua em áreas urbanas e rurais do Estado de São Paulo, Brasil. Rev Panam Salud Pública 2008; 23:78-84.

7. Pine CM, Adair PM, Petersen PE, Douglass C, Burnside G, Nicoll AD, et al. Developing explanatory models of health inequalities in childhood dental caries. Community Dent Health 2004; 21(1 Suppl):86-95.

8. Harris R, Nicoll AD, Adair PM, Pine CM. Risk factors for dental caries in young children: a systematic review of the literature. Community Dent Health 2004; 21:71-85.

9. Beighton D, Brailsford S, Samaranayake LP, Brown JP, Ping FX, Grant-Mills D, et al. A multi-country comparison of caries-associated microflora in demographically diverse children. Community Dent Health 2004; 21(1 Suppl):96-101.

10. Ministério da Saúde. Projeto SB Brasil 2003: condições de saúde bucal da população brasileira 20022003. Resultados principais. Brasília: Ministério da Saúde; 2004.

11. Coordenação Nacional de Saúde Bucal, Secretaria de Atenção à Saúde, Ministério da Saúde. Diretrizes da Política Nacional de Saúde Bucal. Brasília: Ministério da Saúde; 2004.

12. Antunes JLF, Narvai PC. Políticas de saúde bucal no Brasil e seu impacto sobre as desigualdades em saúde. Rev Saúde Pública 2010; 44:360-5.

13. Skeie MS, Raadal M, Strand GV, Espelid I. The relationship between caries in the primary dentition at 5 years of age and permanent dentition at 10 years of age - a longitudinal study. Int J Paediatr Dent 2006; 16:152-60.

14. Noro LRA, Roncalli AG, Mendes Júnior FIR, Lima KC. A utilização de serviços odontológicos entre crianças e fatores associados em Sobral, Ceará, Brasil. Cad Saúde Pública 2008; 24:1509-16.

15. Martins S. Fatores associados à cárie dentária em pré-escolares: uma abordagem sob a ótica do Programa de Saúde da Família [Dissertação de Mestrado]. São Paulo: Universidade de São Paulo; 2003.

16. Nickel DA, Lima FG, Silva BB. Modelos assistenciais em saúde bucal no Brasil. Cad Saúde Pública 2008; 24:241-6.
17. Melo MMDC, Souza WV, Lima MLC, Couto GBL, Chaves TM. Cárie dentária em pré-escolares: um estudo em áreas cobertas pela estratégia saúde da família do Recife, PE, Brasil. Pesqui Bras Odontopediatria Clín Integr 2010; 10:337-43.

18. Fédération Dentaire Internationale. Global goals for oral health in the year 2000. Int Dent J 1982; 32:74-7.

19. Prefeitura do Recife. Plano municipal de saúde: Recife saudável - inclusão social e qualidade no SUS/2006-2009. Recife: Prefeitura do Recife; 2005.

20. World Health Organization. Oral health surveys: basic methods. 4th Ed. Geneva: World Health Organization; 1997.

21. Frias AC, Antunes JLF, Narvai PC. Precisão e validade de levantamentos epidemiológicos em saúde bucal: cárie dentária na cidade de São Paulo, 2002. Rev Bras Epidemiol 2004; 7:144-54.

22. Oliveira LB, Sheiham A, Bönecker MJS. Exploring the association of dental caries with social factors and nutritional status in Brazilian preschool children. Eur J Oral Sci 2008; 116:37-43.

23. Antunes LF, Peres MA, Mello TRC. Determinantes individuais e contextuais da necessidade de tratamento odontológico na dentição decídua no Brasil. Ciênc Saúde Coletiva 2006; 11:79-87.

24. Peres MA, Latorre MRDO, Sheiham A, Peres KG, Barros FC, Hernandez PG, et al. Determinantes sociais e biológicos da cárie dentária em crianças de 6 anos de idade: um estudo transversal aninhado numa coorte de nascidos vivos no Sul do Brasil. Rev Bras Epidemiol 2003; 6:293-6.

25. Leite ICG, Ribeiro RA. Dental caries in the primary dentition in public nursery school children in Juiz de Fora, Minas Gerais, Brazil. Cad Saúde Pública 2000; 16:717-22.

26. Holm AK. Diet and caries in high-risk groups in developed and developing countries. Caries Res 1990; 24 Suppl 1:44-52.

27. Vanderlei LCM, Silva GAP, Braga JU. Fatores de risco para internamento por diarréia aguda em menores de dois anos: estudo de caso-controle. Cad Saúde Pública 2003; 19:455-63.

28. Paunio P, Rautava P, Helenius H, Alanen P, Sillanpää M. The Finnish Family Competence Study: the relationship between caries, dental health habits and general health in 3-year-old Finnish children. Caries Res 1993; 27:154-60.

29. Schoroth RJ, Moffatt MEK. Determinants of early childhood caries (ECC) in a rural Manitoba community: a pilot study. Pediatr Dent 2005; 27:114-20.

30. Vasconcelos EM. A priorização da família nas políticas de saúde. Saúde Debate 1999; 23:6-19.

31. Moynihan P, Petersen PE. Diet, nutrition and the prevention of dental diseases. Public Health Nutr 2004; 7:201-26.

32. Vadiakas G. Case definition, aetiology and risk assessment of early childhood caries (ECC): a revisited review. Eur Arch Paediatr Dent 2008; 9:114-25.

33. Losso EM, Tavares MCR, Silva JYB, Urban CA. Cárie precoce e severa na infância: uma abordagem integral. J Pediatr 2009; 85:295-300. 
34. Al Ghanim NA, Adenubi JO, Wyne AA, Khan NB. Caries prediction model in pre-school children in Riyadh, Saudi Arabia. Int J Paediat Dent 1998; 8:115-22.

35. Rodrigues CS, Sheiham A. The relationships between dietary guidelines, sugar intake and caries in primary teeth in low income Brazilian 3-yearolds: a longitudinal study. Int J Paediat Dent 2000; 10:47-55.

36. Brandão IMG, Arcieri RM, Sundefeld MLM, Moimaz SAS. Cárie precoce: influência de variáveis sócio-comportamentais e do locus de controle da saúde em um grupo de crianças de Araraquara, São Paulo, Brasil. Cad Saúde Pública 2006; 22:1247-56.

37. Woodward M, Walker ARP. Sugar consumption and dental caries: evidence from 90 countries. Br Dent J 1994; 176:297-302.

38. Aquino RC, Philippi ST. Consumo infantil de alimentos industrializados e renda familiar na cidade de São Paulo. Rev Saúde Pública 2002; 36:655-60.
39. Traebert J, Moreira EAM, Bosco VL, Almeida ICS. Transição alimentar: problema comum à obesidade e à cárie dentária. Rev Nutr 2004; 17:247-53.

40. Menghini G, Steiner M, Thomet E, Roos M, Imfeld T. Caries prevalence in 2-year-old children in the city of Zurich. Community Dent Health 2008; 25:154-60.

41. Tinanoff N, O'Sullivan DM. Early childhood caries: overview and recent findings. Pediat Dent 1997; 19:12-6.

42. Hashim R, Thomson WM, Ayers KM, Lewsey JD, Awad M. Dental caries experience and use of dental services among preschool children in Ajman, UAE. Int J Paediatr Dent 2006; 16:257-62.

43. Baldani MHP, Lopes CMDL, Kriger L, Matsuo T. A odontologia para bebês no Estado do Paraná, Brasil - perfil do Programa de Atenção Precoce à Saúde Bucal. JBP, J Bras Odontopediatr Odontol Bebê 2003; 6:210-6.

Recebido em 30/Mai/2010

Versão final reapresentada em 15/Nov/2010

Aprovado em 23/Dez/2010 